Journal of Mathematics and Statistics 3 (4): 207-210, 2007

ISSN 1549-3644

(C) 2007 Science Publications

\title{
On a Class of Nonhomogeneous Fields in Hilbert Space
}

\author{
Raéd Hatamleh \\ Department of Mathematics, Irbid National University, Irbid-Jordan
}

\begin{abstract}
Two-parametric semigroups of operators in Hilbert space with bounded infinitesimal doubly commuting operators are studied. The characteristics describing deviation of a semigroup from unitary one, when infinitesimal operators are unitary, in particular, nonunitary index, have been introduced. Necessary and sufficient conditions for nonunitary index finiteness have been obtained.
\end{abstract}

Keywords: Nonhomogeneous Fields, Multi-parametric Semigroup, Doubly Commuting Operators

\section{INTRODUCTION}

One-parametric semigroups of operators were studied adequately, both from theoretical and applied pointviews ${ }^{[1]}$, A few works in harmonic analysis are devoted to study multi-parametric semigroups ${ }^{[2,3]}$. We study the nonhomogeneous field $u\left(x_{1}, x_{2}\right)$ in Hilbert space $H$ which is presented in the form

$u\left(x_{1}, x_{2}\right)=e^{i x_{1} T_{1}+i x_{2} T_{2}} u_{0}$,

where $u_{0} \in H, T_{1}$ and $T_{2}$ are bounded doubly commuting operators ${ }^{[4]}$. Consider a scalar product

$\left\langle u\left(x_{1}, x_{2}\right), u\left(y_{1}, y_{2}\right)\right\rangle_{H}=K\left(x_{1}, y_{1} ; x_{2}, y_{2}\right)$.

Then if $T_{j}=T_{j}^{*}(j=1,2)$, the function $K\left(x_{1}, y_{1} ; x_{2}, y_{2}\right)$ depends only on corresponding differences $K\left(x_{1}-y_{1} ; x_{2}-y_{2}\right)$ and the field is homogenous.

If $T_{1} \neq T_{1}^{*}$ or $T_{2} \neq T_{2}^{*}$ or both operators $T_{j}(j=1,2)$ are non self-adjoint operators, then the field $u\left(x_{1}, x_{2}\right)$ is nonhomogeneous. In addition, if $T_{j}(j=1,2)$ belongs to a certain class of non selfadjoint operators, one may invoke spectral theory of doubly commuting non self-adjoint operators to study the field $u\left(x_{1}, x_{2}\right)$.

Functional Characteristic of the Nonhomogeneous Field: Consider the case when $T_{j}(j=1,2)$ are doubly commuting unitary or quasi-unitary operators and introduce some numerical and functional characteristics, describing deviation of the field in the form

$u\left(x_{1}, x_{2}\right)=e^{i x_{1} T_{1}+i x_{2} T_{2}} u_{0}$,

where $T_{j}$ are unitary operators. Note that for unitary doubly commuting operators (we call the corresponding field to be unitary) function $K\left(x_{1}, y_{1} ; x_{2}, y_{2}\right)$ may be presented in the form

$$
\begin{aligned}
& K\left(x_{1}-y_{1} ; x_{2}-y_{2} ; x_{1}+y_{1} ; x_{2}+y_{2}\right)= \\
& \int_{0}^{2 \pi} e^{i\left(x_{1}-y_{1}\right) \cos f_{1}(\lambda)+i\left(x_{2}-y_{2}\right) \cos f_{2}(\lambda)} \\
& \times e^{-\left(x_{1}+y_{1}\right) \sin f_{1}(\lambda)-\left(x_{2}+y_{2}\right) \sin f_{2}(\lambda)} d F_{\lambda},
\end{aligned}
$$

where, $\quad f_{k}(\lambda)$ real-value functions, $\Delta F_{\lambda}=\left\langle\Delta E_{\lambda} u_{0}, u_{0}\right\rangle$,

and $E_{\lambda}$ is the spectral function of unitary operator $T_{0}=\int_{0}^{2 \pi} e^{i \lambda} d E_{\lambda}$.

The above form of $K$ follows from the Neuman theorem for generating operator $T_{0}$ of a set of mutually commuting selfadjoint (unitary) operators ${ }^{[5]}$.

Taking into the account the well-known fact for commuting operators $T_{1}$ and $T_{2}$ one of them is a function of another ${ }^{[5]}$. It is not difficult to verify that if $T_{1}$ and $T_{2}$ are the unitary commutative operators then the function $K\left(x_{1}, y_{1} ; x_{2}, y_{2}\right)$ satisfies the following equation

$$
L_{x_{j} y_{j}} K\left(x_{1}, y_{1} ; x_{2}, y_{2}\right)=0, \quad(j=1,2)
$$


where

$L_{x y}=I-\frac{\partial^{2}}{\partial x \partial y}$.

From the applied point of view $K\left(x_{1}, y_{1} ; x_{2}, y_{2}\right)$ is the correlation function for some random field, because $K\left(x_{1}, y_{1} ; x_{2}, y_{2}\right)$ is Hermitian nonnegative function. Hence there exists Gaussian normal field for which $K\left(x_{1}, y_{1} ; x_{2}, y_{2}\right)$ is the correlation function and the results obtained may be interpreted as a correlation theory for nonhomogeneous random field. Here after we will consider that

$$
H=H_{u}=\overline{\bigvee_{x_{1}, x_{2} \geq 0} T^{x_{1}} T^{x_{2}} u_{0}}, \quad\left(x_{j} \text { are integers }\right) \text {. }
$$

Let us consider the field

$u^{*}\left(x_{1}, x_{2}\right)=e^{i x_{1} T_{1}^{*}+i x_{2} T_{2}^{*}} u_{0}$,

which, henceforth, we will call it the adjoint field.

It is obvious that for the field $e^{-i x_{1} T_{1}+i x_{2} T_{2}} u_{0}\left(T_{1}\right.$ and $T_{2}$ double commuting operators ) to be unitary it is necessary and sufficient that $K$ should be in accordance with

$L_{x_{j} y_{j}} K\left(x_{1}, y_{1} ; x_{2}, y_{2}\right)=0, \quad(j=1,2)$

Lemma 1: Let $H_{u}=H_{u}^{*}=H$, and $u\left(x_{1}, x_{2}\right)=e^{i x_{1} T_{1}+i x_{2} T_{2}} u_{0}$. Then the necessary and sufficient for $T_{1}$ and $T_{2}$ to be commutative is that

$\frac{\partial^{2}}{\partial x_{1} \partial y_{2}} \widetilde{K}\left(x_{1}, y_{1} ; x_{2}, y_{2}\right)=$

$\frac{\partial^{2}}{\partial x_{2} \partial y_{1}} \widetilde{K}\left(x_{1}, y_{1} ; x_{2}, y_{2}\right)$

where

$\widetilde{K}\left(x_{1}, y_{1} ; x_{2}, y_{2}\right)=\left\langle u\left(x_{1}, x_{2}\right), u^{*}\left(y_{1}, y_{2}\right)\right\rangle$.

The lemma proof follows from the definition of the function, $\widetilde{K}\left(x_{1}, y_{1} ; x_{2}, y_{2}\right)$ and a relationship $\frac{\partial^{2} \widetilde{K}}{\partial x_{\ell} \partial y_{m}}=-\left\langle T_{\ell} T_{m} u\left(x_{1}, x_{2}\right), u^{*}\left(y_{1}, y_{2}\right)\right\rangle$.

If $L_{x_{1} y_{1}} L_{x_{2} y_{2}} K\left(x_{1}, y_{1} ; x_{2}, y_{2}\right) \neq 0$, then the function

$W\left(x_{1}, y_{1} ; x_{2}, y_{2}\right)=L_{x_{1} y_{1}} L_{x_{2} y_{2}} K\left(x_{1}, y_{1} ; x_{2}, y_{2}\right)$ may be considered as a functional characteristic of deviation infinitesimal commutative operators $T_{1}$ and $T_{2}$ from unitary operators.

If $T_{1}$ and $T_{2}$ are doubly commuting operators $\left(\left(\left[T_{1}, T_{2}\right]=0,\left[T_{1}, T_{2}^{*}\right]=0\right)\right.$, then from (3) we may obtained the following presentations for $W$ :

$W\left(x_{1}, y_{1} ; x_{2}, y_{2}\right)=$

$\left\langle\left(I-T_{1}^{*} T_{1}\right)\left(I-T_{2}^{*} T_{2}\right) u\left(x_{1}, x_{2}\right), u\left(y_{1}, y_{2}\right)\right\rangle$.

The presentation (4) is significant for further studies.

Remark 1: To reconstruct $K\left(x_{1}, y_{1} ; x_{2}, y_{2}\right)$ by $W\left(x_{1}, y_{1} ; x_{2}, y_{2}\right)$ one may solve Darboux-Goursat problem for equation

$L_{x_{1} y_{1}} L_{x_{2} y_{2}} K\left(x_{1}, y_{1} ; x_{2}, y_{2}\right)=W\left(x_{1}, y_{1} ; x_{2}, y_{2}\right)$ twice, and defining appropriate conditions additionally.

Remark 2: If the operators $T_{1}$ and $T_{2}$ are commuting operators, but are not doubly commuting, then $W\left(x_{1}, y_{1} ; x_{2}, y_{2}\right)=$ $\left\langle\left(I-T_{1}^{*} T_{1}-T_{2}^{*} T_{2}+T_{2}^{*} T_{1}^{*} T_{1} T_{2}\right) u\left(x_{1}, x_{2}\right)\right.$,

$\left.u\left(y_{1}, y_{2}\right)\right\rangle$

and further analysis is based on assumption of commutant $\left[T_{1}, T_{2}^{*}\right]$ properties, for example $T_{1}, T_{2}^{*}$ and $\left[T_{1}, T_{2}^{*}\right]$ form Lie algebra.

Theorem 1: If $\operatorname{dim} H_{0}=r<\infty$, where $H_{0}=\overline{\left(I-T_{1}^{*} T_{1}\right)} H \cap \overline{\left(I-T_{2}^{*} T_{2}\right)} H$, then

$W\left(x_{1}, y_{1} ; x_{2}, y_{2}\right)=\sum_{\alpha=1}^{r} \lambda_{\alpha} \Phi_{\alpha}\left(x_{1}, x_{2}\right) \overline{\Phi_{\alpha}\left(y_{1}, y_{2}\right)}$,

where $\Phi_{\alpha}\left(x_{1}, x_{2}\right)=\left\langle u\left(x_{1}, x_{2}\right), h_{\alpha}\right\rangle, h_{\alpha} \in H_{0}$, and $\lambda_{\alpha}$ are real numbers.

Proof: Consider the orthonormal basis $\left\{h_{\alpha}\right\}_{\alpha=1}^{r}$ in $H_{0}$, consisting of eigenvector contraction of self-adjoinet operator $\left(I-T_{1}^{*} T_{1}\right)\left(I-T_{2}^{*} T_{2}\right)$ onto its invariant subspace $H_{0}$. Since 


$$
\begin{aligned}
B_{H} & =\left(I-T_{1}^{*} T_{1}\right)\left(I-T_{2}^{*} T_{2}\right) u\left(x_{1}, x_{2}\right) \\
& =\sum_{\alpha=1}^{r}\left\langle B u\left(x_{1}, x_{2}\right), h_{\alpha}\right\rangle h_{\alpha} \\
& =\sum_{\alpha=1}^{r}\left\langle u\left(x_{1}, x_{2}\right), B h_{\alpha}\right\rangle h_{\alpha} \\
& =\sum_{\alpha=1}^{r} \lambda_{\alpha}\left\langle u\left(x_{1}, x_{2}\right), h_{\alpha}\right\rangle h_{\alpha},
\end{aligned}
$$

where $B h_{\alpha}=\lambda_{\alpha} h_{\alpha}$ and $\lambda_{\alpha}$ are eigenvalues of the operator $B$.

As a result, we obtain

$W\left(x_{1}, y_{1} ; x_{2}, y_{2}\right)=$

$\sum_{\alpha=1}^{r} \lambda_{\alpha} \Phi_{\alpha}\left(x_{1}, x_{2}\right) \overline{\Phi_{\alpha}\left(y_{1}, y_{2}\right)}$.

Remark, that the function $K\left(x_{1}, y_{1} ; x_{2}, y_{2}\right)$ defines the Hilbert-valued function $u\left(x_{1}, x_{2}\right)$ quite completely. The next assertion is valid.

Lemma 2: Consider the two functions $u_{1}\left(x_{1}, x_{2}\right)$ and $u_{2}\left(x_{1}, x_{2}\right)$ with values belonging to the Hilbert spaces

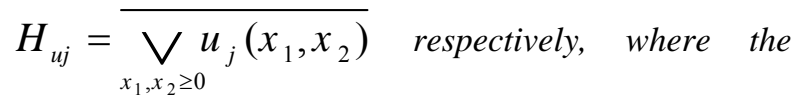
scalar product is generated by the respective function $K\left(x_{1}, y_{1} ; x_{2}, y_{2}\right)=\left\langle u_{j}\left(x_{1}, x_{2}\right), u_{j}\left(y_{1}, y_{2}\right)\right\rangle_{H_{j}}$ $=K_{j}\left(x_{1}, y_{1} ; x_{2}, y_{2}\right)$.

If $K_{1}\left(x_{1}, y_{1} ; x_{2}, y_{2}\right)=K_{2}\left(x_{1}, y_{1} ; x_{2}, y_{2}\right)$, then there exists a unitary transformation $U \in\left[H_{1}, H_{2}\right]$ such that $u_{2}\left(x_{1}, x_{2}\right)=U u_{1}\left(x_{1}, x_{2}\right)$. Moreover if $u\left(x_{1}, x_{2}\right)=e^{i x_{1} T_{1}+i x_{2} T_{2}} u_{0_{1}}$, then $u_{2}\left(x_{1}, x_{2}\right)$ is also generated by two-parametric semigroup of operators $u_{2}\left(x_{1}, x_{2}\right)=e^{i x_{1} B_{1}+i x_{2} B_{2}} u_{0_{2}}$.

Proof: Consider lineals

$L_{j}=\left\{\sum_{\alpha, \beta=1}^{n_{1}, n_{2}} C_{\alpha, \beta} u_{j}\left(x_{\alpha}, x_{\beta}\right)\right\} n_{1}, n_{2}<\infty$,

where, $C_{\alpha, \beta}$ are complex numbers. For $h_{1}^{(j)}, h_{2}^{(j)} \in L_{j}$ define binary form $\left\langle h_{1}^{(j)}, h_{2}^{(j)}\right\rangle_{L_{j}}=$

$\sum_{\alpha, \beta=1}^{n_{1}, n_{2}} \sum_{p, q=1}^{m_{1}, m_{2}} C_{\alpha, \beta} Q_{p, q} K_{j}\left(x_{\alpha}, y_{p} ; x_{\beta}, y_{q}\right)$,

where,

$$
\begin{aligned}
& h_{1}^{(j)}=\sum_{\alpha, \beta=1}^{n_{1}, n_{2}} C_{\alpha, \beta} u_{j}\left(x_{\alpha}, x_{\beta}\right), \\
& h_{2}^{(j)}=\sum_{p, q=1}^{m_{1}, m_{2}} Q_{p, q} u_{j}\left(x_{p}, x_{q}\right) .
\end{aligned}
$$

Then $L_{j}$ become pre-Hilbert spaces. Define isometric (by virtue of equality $\left.K_{1}\left(x_{1}, y_{1} ; x_{2}, y_{2}\right)=K_{2}\left(x_{1}, y_{1} ; x_{2}, y_{2}\right)\right)$, transformation of $L_{1}$ into $L_{2}$ :

$U\left(\sum_{\alpha, \beta=1}^{n_{1}, n_{2}} C_{\alpha, \beta} u_{1}\left(x_{\alpha}, x_{\beta}\right)\right)$
$=\left(\sum_{\alpha, \beta=1}^{n_{1}, n_{2}} C_{\alpha, \beta} u_{2}\left(x_{\alpha}, x_{\beta}\right)\right)$.

Extending $U$ for closures $L_{1}$ and $L_{2}$ we get the first assertion of the Lemma. The second part of the Lemma follows immediately from the evident relationships:

$u_{2}\left(x_{1}, x_{2}\right)=U u_{1}\left(x_{1}, x_{2}\right)=$ $U e^{i x_{1} T_{1}+i x_{2} T_{2}} u_{0_{1}}=e^{i x_{1} B_{1}+i x_{2} B_{2}} u_{0_{2}}$,

where $B_{j}=U T_{j} U^{-1}, u_{0_{2}}=U u_{0_{1}}$.

Nonunitary index: Let us now define a numerical characteristic for the field deviation from the unitary field. Let us call the nonunitary index the maximal rank of quadratic forms

$$
\sum_{\ell, m=1}^{n} W\left(x_{1}^{(\ell)}, y_{1}^{(\ell)} ; x_{2}^{(m)}, y_{2}^{(m)}\right) Z_{\ell} \bar{Z}_{m}, \quad n \leq \infty .
$$

For the unitary field a nonunitary property coefficient is equal to 0 , since $W\left(x_{1}, y_{1} ; x_{2}, y_{2}\right)=0$.

Theorem 2: In order that the field $u\left(x_{1}, x_{2}\right)=e^{i x_{1} T_{1}+i x_{2} T_{2}} u_{0}$, has a finite nonunitary index it is necessary and sufficiently that dim $H_{0}=r<\infty$, where $T_{1}$ and $T_{2}$ are doubly commuting operators and 
$u_{0} \in H_{0}=\overline{\left(I-T_{1}^{*} T_{1}\right) H} \cap \overline{\left(I-T_{2}^{*} T_{2}\right) H}$.

Proof:

Sufficiency: When $\operatorname{dim} H_{0}=r<\infty$, there exists representation (5) for $W\left(x_{1}, y_{1} ; x_{2}, y_{2}\right)$ and

$\sum_{\ell, m=1}^{n} W\left(x_{1}^{(\ell)}, y_{1}^{(\ell)} ; x_{2}^{(m)}, y_{2}^{(m)}\right) Z_{\ell} \bar{Z}_{m}=\sum_{v=1}^{r} \lambda_{v}\left|\zeta_{v}\right|^{2}$,

where $\zeta_{v}=\sum_{\ell=1}^{n} \Phi_{v}\left(x_{1}^{(\ell)}, x_{2}^{(\ell)}\right) Z_{\ell}$. It follows that the rank of quadratic form does not exceed $r$.

Necessity: Let us consider the sequence of pares of real numbers

$x_{\ell}=\left(x_{1}^{(\ell)}, x_{2}^{(\ell)}\right),(\ell=\overline{1, n})$.

Then

$\sum_{\ell, m=1}^{n} W\left(x_{\ell}, x_{m}\right) Z_{\ell} \bar{Z}_{m}=\left\langle\left(I-T_{1}^{*} T_{1}\right)\left(I-T_{2}^{*} T_{2}\right) h, h\right\rangle$

where $h=\sum_{\ell=1}^{n} Z_{\ell} u\left(x_{1}^{(\ell)}, x_{2}^{(\ell)}\right)$.

Let

$H_{n}=\left\{h: h=\sum_{\ell=1}^{n} Z_{\ell} u\left(x_{1}^{(\ell)}, x_{2}^{(\ell)}\right)\right\}, H_{n} \subset H_{u}$.

Consider the subspace $G_{n}=P_{n}\left(I-T_{1}^{*} T_{1}\right)\left(I-T_{2}^{*} T_{2}\right) P_{n} H_{u}$, where $P_{n}$ is the projection operator onto subspace $H_{n}$. It is obvious that $G_{n} \subseteq P_{n} H_{0}$ and the rank of form $\sum_{\ell, m=1}^{n} W\left(x_{\ell}, x_{m}\right) Z_{\ell} \bar{Z}_{m}$ is equal to $\operatorname{dim} G_{n}$. It is evident that $H_{1} \subset H_{2} \subset \ldots \subset H_{n} \subset \ldots \quad$ and $\lim _{n \rightarrow \infty} P_{n}=I$, hence $\operatorname{rank} W>\operatorname{dim} G_{n}$ and $\operatorname{rank} W \geq \lim _{n \rightarrow \infty} G_{n}=\operatorname{dim} H_{0}$. This implies that rank $\operatorname{dim} H_{0} \leq r$.

Similarly one may prove the next theorem.
Theorem 3: In order that the field $u\left(x_{1}, x_{2}\right)=e^{i x_{1} T_{1}+i x_{2} T_{2}} u_{0}$,

has a finite nonunitary index it is necessary and sufficient that the subspaces

$H_{0}^{(j)}=\overline{\left(I-T_{j}^{*} T_{j}\right) H} \quad(j=1,2)$

be finite-dimensional where, $u_{0} \in H, T_{j}$ are doubly commuting operators.

Further development of suggested approach is related to the spectral theory for the doubly commuting contraction systems and their triangular and universal models ${ }^{[6]}$. Thus, one may derive canonical representation for $W\left(x_{1}, y_{1} ; x_{2}, y_{2}\right)$ and perform harmonic analysis of two-parametric semigroups $e^{i x_{1} T_{1}+i x_{2} T_{2}}$ when $T_{1}$ and $T_{2}$ are doubly commuting contractions.

\section{REFERENCES}

1. Hille, E., Phillips, R.S., 1957. Functional Analysis and Semi-Groups, Providence, 829.

2. Livčic, Moshe S.,Waksman, Leonid L., 1987. Commuting Nonself-adjoint Operator in Hilbert Space, Lect Notes Math, \#1272, ed. Coic and E.Ecumann, 115.

3. Livčic, M. S., Kravitsky, N., Markus, A., Vinnikov, V., 1995. Theory of Commuting Nonself-adjoint Operators, Kluver academic publ., Dordrent - London, 332.

4. Zolotarev, V., 1997. Functional Models for Algebras of Linear Nonself-adjoint Operators, Zeit fur Ang. Math and Mech., 77(2): 695-696.

5. Riesz, F., Sz.-Nagy, B., 1972. Lessons D'Analyse Fonctionnelle, Akademiani Kiodo, Budapest, 587.

6. Zolotarev, V.,1976. Triangular Models of Systems of Doubly Commuting Operators (Russian), Akad. Nauk Armjan SSR Dokl 63,136-140 (Reviewer J.L. Looper) 47A 45. 